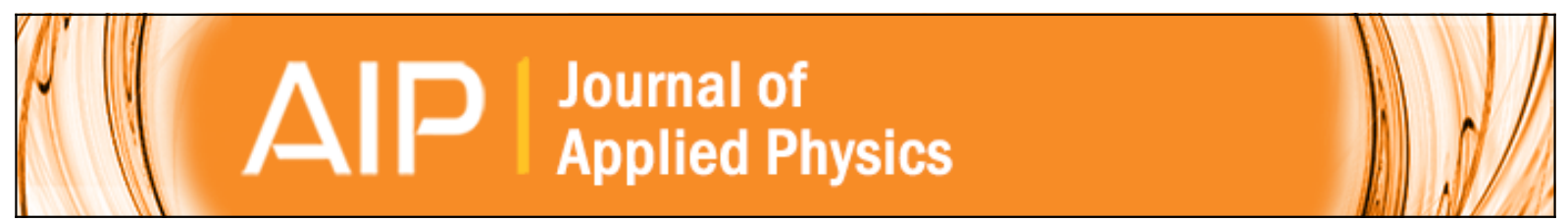

Theory of electrical characteristics for metaloxideinsulator Schottky barrier and metal insulatormetal structures

H. M. Gupta and Marta B. Morais

Citation: Journal of Applied Physics 68, 176 (1990); doi: 10.1063/1.347111

View online: http://dx.doi.org/10.1063/1.347111

View Table of Contents: http://scitation.aip.org/content/aip/journal/jap/68/1?ver=pdfcov

Published by the AIP Publishing

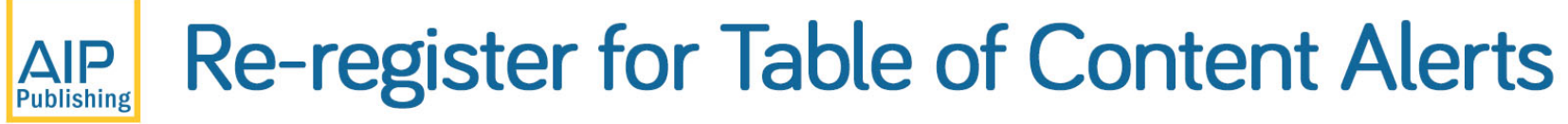

Create a profile.

Sign up today! 


\title{
Theory of electrical characteristics for metal-oxide-insulator Schottky barrier and metal-insulator-metal structures
}

\author{
H. M. Gupta \\ Departamento de Fisica IGCE, UNESP, C. P. 178, 13500 Rio Claro SP, Brazil \\ Marta B. Morais \\ Departamento de Ciências, Faculdade de Engenharia, UNESP, 15378 Ilha Solteira SP, Brazil
}

(Received 14 August 1989; accepted for publication 13 February 1990)

\begin{abstract}
The metal-insulator or metal-amorphous semiconductor blocking contact is still not well understood. Here, the intimate metal-insulator and metal-oxide-insulator contact are discussed. Further, the steady-state characteristics of metal-oxide-insulator-metal structures are also discussed. Oxide is an insulator with wider energy band gap (about $50 \AA$ thick). A uniform energetic distribution of impurities is considered in addition to impurities at a single energy level inside the surface charge region at the oxide-insulator interface. Analytical expressions are presented for electrical potential, field, thickness of the depletion region, capacitance, and charge accumulated in the surface charge region. The electrical characteristics are compared with reference to relative densities of two types of impurities. In $I$ is proportional to the square root of applied potential if energetically distributed impurities are relatively important. However, distribution of the electrical potential is quite complicated. In general energetically distributed impurities can considerably change the electrical characteristics of these structures.
\end{abstract}

\section{INTRODUCTION}

The metal-semiconductor (MS) interface has played an important role in many technological developments. ${ }^{1-3}$ However, a full understanding of the physics behind the origin and electrical properties of the Schottky barriers is not yet properly understood from a theoretical point of view in spite of many works, ${ }^{4-8}$ even for crystalline materials. The metal-insulator contact, particularly that of amorphous material, is an even more complicated problem because of the uncertainty of the nature and distribution of impurities at contact. Recently many authors ${ }^{9-12}$ have discussed the metal-amorphous-silicon contact because of its technological importance.

Electrical conduction through metal-insulator-metal (MIM) structures has been the subject of intensive theoretical and experimental investigation in the last three decades. ${ }^{13-14}$ In very thin insulating films $(<50 \AA)$, tunneling from metal to metal is a predominant charge conduction mechanism. ${ }^{15}$ In cases of thick finns ( $>1 \mu \mathrm{m}$ ), bulk properties of insulators are definitely more important. The charge conduction mechanisms in blocking contact MIM structures with intermediate insulator thicknesses ( $1000 \AA$ to 1 $\mu \mathrm{m})$ are still uncertain, while this range of insulator thickness is technologically the most important.

A large number of classical ${ }^{16-17}$ and quantum ${ }^{18}$ mechanical models have been proposed for Schottky barriers. The most classical is the one proposed by Schottky in 1939, which is based on a perfect contact between the metal and semiconductor leading to Fermi-level alignment and band bending to neutralize the charge transfer. In this model, donor-type impurities at a single energy level are considered. ${ }^{4}$

The localized impurities are distributed throughout an amorphous material..$^{13}$ The importance of surface states and energetically distributed impurities at interface in MIS or MS Schottky barriers are well recognized. ${ }^{7}$ However, most of the recent work is on either formation of the Schotky barrier and relation of barrier height to these impurity distributions, ${ }^{6-19}$ or on the determination of energetic distribution of these impurities in amorphous materials, ${ }^{9-11}$ particularly amorphous silicon through the capacitance-voltage relationship. Mostly numerical calculations are done to obtain energy distribution of these impurities. Tarasenwicz and Salama ${ }^{20}$ discussed the theory of the surface depletion region for a semiconductor with linearly graded impurities at a single energy level, which is important for ion-implanted devices.

The importance of energetically distributed impurities on variations of electrical field and potential within the depletion region and on electrical characteristics of metal-insulator-metal structures with blocking contacts is not discussed in detail; particularly, no analytical approach is made. For detalled discussion of steady and nonsteady-state electrical characteristics of MIM structures, impurities at a single energy level are usually considered. ${ }^{4,21-23}$ In the present paper we discuss the effects of energetically distributed impurities on electrical characteristics of Schottky barriers and MIM structures. We found that impurities can considerably change the electrical characteristics of these structures.

\section{I1. METAL-SEMTCONDUCTOR CONTACT}

Schottiky ${ }^{4,16}$ considered a uniform positive charge density in the depletion region and intimate metal-semiconductor contact. When the external contact potential $\left(V_{c}\right)$ equals zero, the general shapes of energy-band levels are as shown in Fig. $1(\mathrm{a}), E_{\mathrm{c}}$ is the energy of the bottom of the conduction band, $E_{f}$ is the Fermi energy. $\psi_{\mathrm{bi}}$ is an internal built-in potential on the barrier and is equal to the difference of metal and insulator work functions $\left(\psi_{b i}=\psi_{m}-\psi_{i}\right)$. $\phi_{b}$ is the energy barrier for metal electrodes. The thickness of the depletion layer $\left(\lambda_{0}\right)$ is given by 


$$
\lambda_{0}=\left(2 k \epsilon_{0} \psi_{\mathrm{bi}} / q^{2} N_{\imath}\right)^{1 / 2},
$$

where $K$ is the dielectric constant, $\epsilon_{0}$ is the permittivity of free space, and $g N$, is the positive charge density in the depletion.

When the external contact potential equals $V_{c}$, the shape of Fermi energy in the depletion layer is uncertain and is normaly not drawn. ${ }^{4,5,24}$ As the surface states at the metalsemiconductor interface are in intimate contact with metal states, we expect that those states which are below the metal Fermi level continue to remain filled up. Thus the expected Fermi level in the insulator is as shown in Fig. 1(b). This means that during the semiconductor (insulator in case of MIM structures) relaxation, the electrons flow from the bulk impurity states in region $A B$ of Fig. $1(b)$ and thus growth of the cathodic depletion region takes place. The positive charge density in the depletion region does not change significantly with applied contact potential even in case of energetically distributed impurities. The thickness of the depletion layer $\left[\lambda\left(V_{c}\right)\right]$, considering uniform positive charge density $\left(q N_{2}\right)$, is given by

$$
\lambda\left(V_{c}\right)=\left(2 R \epsilon_{0} q V_{c} / q^{2} N_{t}\right)^{1 / 2}
$$

where $q V_{c}=\left(q V_{\text {ext }}+\psi_{b i}\right) V_{\text {ext }}$ is the external contact potential.

Energy-band levels for metal-insulator-semiconductor structures for an applied contact potential equal to zero and $V_{c}$ are shown in Figs. 2(a) and $2(b)$, respectively. At contact potential $V_{c}$, the Fermi energy in relation to $E_{c}$ comes down and therefore some of the states which were filled up at $V_{c}=0$ are now empty. This means that the positive charge density in the depletion region increases with the applied
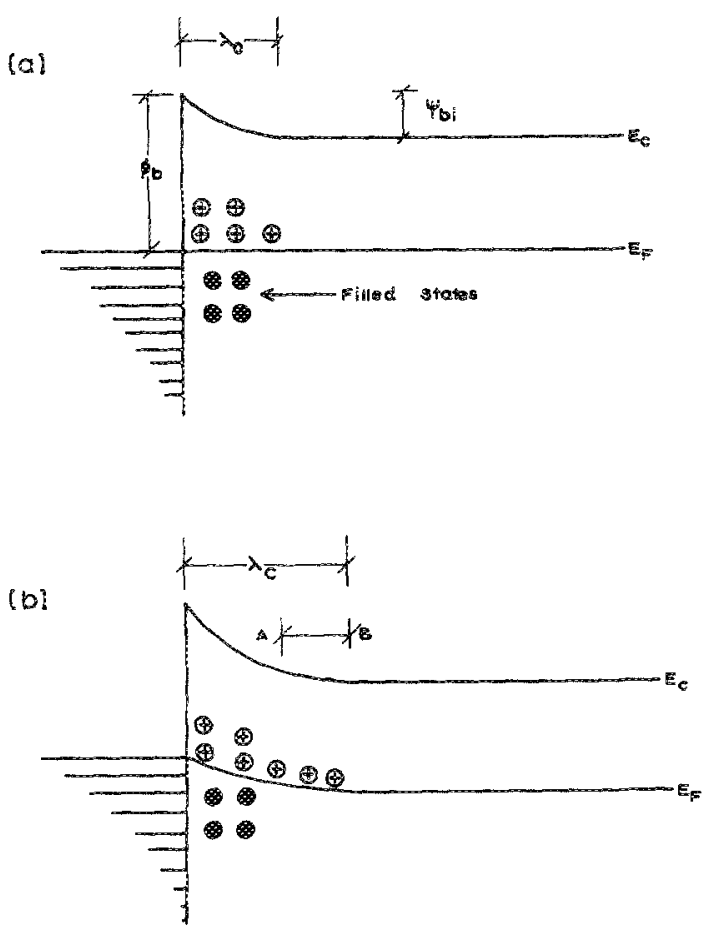

FIG. E. Energy-band levels at blocking metal-semiconductor (also meta:insulator) contact (a) before applying contact potential, and (b) after appiying an externai contact potential. (a)
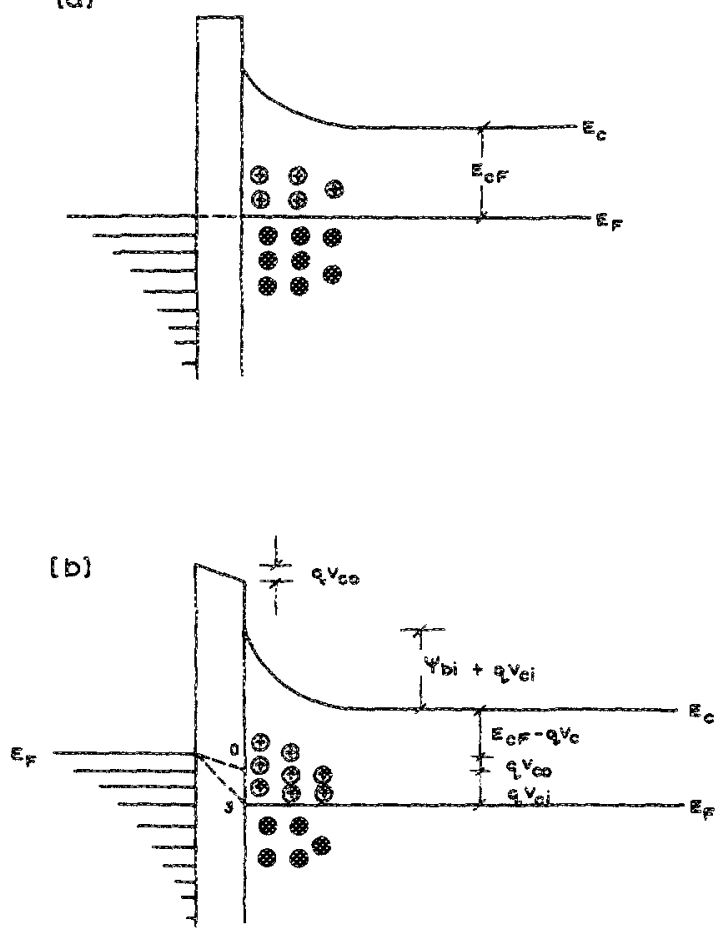

FIG. 2. Energy-band levels at blocking metal-oxide-semiconductor (aiso metal-oxide-insulator) contac (a) before applying potential, and (b) after applying an external contact potential.

contact potential and the charges that flow in the semiconductor during its relaxation may come from the energetically distributed states. Thus the relative increase in the depletion region is less in comparison to intimate Schottkly contact and the energetically distributed impurities are more important. There are many recent studies ${ }^{25,26}$ on structures close to the metal-semiconductor interface. In general, we can say that an anomalous region exists at or within $1000 \AA$ of the surface, particularly for amorphous materials. Generally a thin insulating layer is considered to be present between metal and semiconductor. ${ }^{5-17,27}$ In the so-called Schoteky barrier, this layer is very thin $(\cong 10 \AA)$, so that surface states at the insulator-semiconductor interface are in intimate contact with the metal. ${ }^{17}$ These contacts are made by cleaving the semiconductor surface in an ultrahigh vacuum so as to create a fresh surface and then immediately evaporating the metal. In many cases, particularly of amorphous semiconductors or insulators, the thickness of this insulating layer (we denote this layer as an oxide layer in MIM structures) can be of the order of $50 \AA$ and thus interface states are no longer in intimate contact with metal states through tunneling. Thus we can have, in fact, metal-insulator-semiconductor contact. In metal-insulator-metal structures we may have metal-oxide-insulator contacts instead of intimate metal-insulator contacts, where oxide is an insulator of wider energy band gap. In this case our structure is metaloxide-insulator-oxide-metal. As the anode is forward biased and offers very little resistance to current, we continue to consider it as intimate insulator-metal contact. As such our structure is metal-oxide-insulator-metal (MOIM) as shown in Fig. 3. 


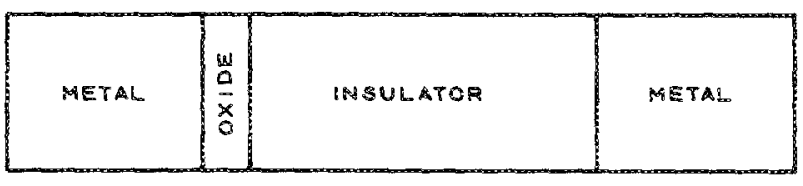

FIG. 3. Metal-oxide-insulator-metal structure.

Many authors ${ }^{9-12}$ have discussed capacitance versus applied potential characteristics of metal-amorphous semiconductor contact (particularly amorphous silicon) to know impurity distribution in semiconductors. They considered quasi-Fermi level to be flat throughout the depletion region, which appears to be an incorrect assumption for intimate metal-semiconductor contact. We think that this assumption is valid only for metal-insulator-semiconductor-zype structures with an insulator thickness of the order of $50 \AA$ or more. This assumption needs more discussion. ${ }^{28}$ However, if the Fermi level is flat throughout the depletion layer for an applied potential even for intimate metai-insulator contacts we still can use the results here except that, in this case, $V_{\text {ox }}$ is zero.

\section{FOAMULATIOA}

We are considering a metal-oxide-insulator contact at interface. The thickness of the oxide layer is about $50 \AA$, so that oxide-insulator states are no longer in contact with metal states. The oxide layer is considered to be free from any kind of electrical charge. This means that the electrical field is constant throughout the oxide layer. If the oxide layer thickness is very small in comparison to the depletion layer thickness, the potential fall across the oxide layer is small and can be neglected. This is normally the case when the effective density of impurity states in the depletion layer is less than $10^{18} / \mathrm{cm}^{3}$.

The impurity states are distributed throughout in prokibited energy bands in an amorphous insulator. Apart from this we have energetically distributed surface states at the oxide-insulator interface. Joining these two types of impurities, the impurity density per $\mathrm{eV}$ at energy $\epsilon$ at distance $x$ from the interface is given by

$$
N(\epsilon, x)=A_{\epsilon}+B_{\varepsilon} \exp (-x / R),
$$

where $A_{\epsilon}, B_{\epsilon}$, and $R$ are constants and depend on the method of fabrication of the device. The influence of a spatially nonhomogeneous trap distribution on the electrical characteristics has been discussed by $\mathrm{KaO}$ and Hwang ${ }^{29}$ and more recently by Rybicki and Chybicki. ${ }^{30}$

In order to study the essential effects of the energetically distributed impurities, we consider $R \gg x$, that is, impurity density is independent of $x$ in the depletion region. Further, we are considering the case when impurities are uniformly distributed throughout the prohibited energy band. Thus $N(\epsilon, x)$ is constant. We denote it by $N_{00}$. Apart from energet:cally distributed impurities, we are considering $N_{d}$ impurities $/ \mathrm{cm}^{3}$ at an energy level $E_{t}$ which is considered to be coinciding with the Fermi level. These impurities can be doped impurities.

As the contact is blocking, we consider that in the re- verse-biased steady state, almost the whole of the applied potential appears across the contact. The total contact potential $\left(V_{c}\right)$ is thus $V_{\mathrm{bi}}+V_{\mathrm{app}}-V_{\mathrm{ox}}$, where $V_{\mathrm{bi}}$ is $\left(\psi_{\mathrm{bi}} / q\right)$. $V_{o x}$ is the potential on the oxide layer, and in the charge-free oxide layer is given by $V_{\mathrm{ox}}=L_{\mathrm{ox}} \times E_{\mathrm{ox}} \cdot L_{\mathrm{ox}}$ is the thickness of the oxide layer, and $E_{0 \mathrm{x}}$ is the electric field in the oxide layer, which is equal to the electric field at the oxide-insulator contact $\left[E_{c}(0)\right]$. Thus

$$
V_{c}=V_{\mathrm{ap}}+V_{\mathrm{bi}}-L_{\mathrm{ox}} E_{c}(0) \text {. }
$$

If the contact potential at distance $x$ from the oxideinsulator interface is $V(x)$, the total positive charge density at this point is

$$
p(x)=q\left[N_{d}-q N_{0} V(x)\right] .
$$

We are considering that contact potential $V(x)$ is $-V_{c}$ at $x=0$ and 0 at $x=W$, where $W$ is the thickness of the depletion layer and $q$ is the magnitude of the electronic charge. In reverse-biased contact, $V(x)$ is negative so that both types of impurities are creating a positive charge.

\section{CALCULATIONS}

The distribution of electric potential and field is obtained through the solution of Poisson's equation, which in the present case is

$$
\frac{d^{2} V}{d x^{2}}=-\frac{q}{K \epsilon_{0}}\left[N_{d}-q N_{0} V(x)\right] .
$$

Integrating Eq. (6) with the boundary conditions (i) for $x=0, V$ is $-V_{c}$ and (ii) for $x=w_{v} v$ and electrical field $(E)$ are zero, we get

$$
\frac{d V}{d x}=\sqrt{K_{1} V^{2}-2} \overline{K_{2}} \bar{V},
$$

where

$$
K_{1}=q_{2}^{2} N_{0} / K \epsilon_{0}
$$

and

$$
K_{2}=q N_{d} / K \epsilon_{\mathrm{c}} .
$$

Integrating Eq. (7) with the boundary conditions and using $v_{s}=-v$, that is $v_{s}$ gives the magnitude of the reversed biased potential, we get

$$
v_{s}=\frac{K_{2}}{K_{1}}\left[\cosh \left(\cosh \frac{\left(K_{1} V_{c}+K_{2}\right)}{K_{2}}-\sqrt{K_{1}} \cdot x\right)-1\right]
$$

Equation (9) gives the variation of the contact potential inside the depletion layer in terms of the total contact potential on the depletion region $\left(V_{c}\right)$ considering $V_{s}=0$, for $x=W$, the thickness of the depletion layer is given as

$$
W=1 / \bar{K}_{1} \cosh ^{-1}\left[\left(K_{1} V_{c}+K_{2}\right) / K_{2}\right] .
$$

Using this resuit, Eq. (9) simplifies to

$$
V_{s}=\left(K_{2} / K_{1}\right)\left\{\cosh \left[\sqrt{k_{1}}(W-x)\right]-1\right\} \text {. }
$$

Differentiating Eq. (11) with respect to $x$, the magnitude of the electrical field in terms of $W$ or $V_{c}$ is given by

$$
E_{c}(x)=-\frac{d V_{s}}{d x}=\frac{K_{2}}{\sqrt{k_{1}}} \sinh \left[\sqrt{k_{1}}(W-x)\right] .
$$


$W$ is related to $V_{c}$ through Eq. (10). The electrical field at the oxide-insulator interface $(x=0)$ is

$$
E_{c}(0)=\left(K_{2} / \sqrt{k_{1}}\right) \sinh \left(\sqrt{k_{1}} W\right)
$$

The potential fall across the oxide layer is thus

$$
V_{\mathrm{ox}}=L_{\mathrm{ox}}\left(k_{2} / \sqrt{k_{1}}\right) \sinh \left(\sqrt{k_{1}^{-}} W\right) .
$$

The value of $E_{c}(x)$ for a given $V_{\text {ap }}$ is obtained by solving Eqs. (4), (10), (12), and (13) simultaneously.

The electrical charge $(Q)$ inside the depletion region is

$$
Q=\int_{0}^{w} \rho(x) d x=\int_{0}^{w} q\left(N_{d}+q N_{0} V_{s}\right) d x
$$

Using Eq. (11) for $V_{s}$ and integrating, we finally get

$$
Q=\left(q N_{d} / \sqrt{k_{1}}\right) \sinh \left(\sqrt{k_{1}} W\right) \text {. }
$$

Through Eqs. (13) and (15), it is easy to see that

$$
Q=K \epsilon_{0} E_{\mathrm{c}}(0)
$$

This is in accordance with Poisson's equation. The capacitance of the depletion region can be calculated through

$$
C=\frac{d Q}{d V_{\mathrm{ap}}}=\frac{d Q}{d V_{c}} \frac{d V_{c}}{d V_{\mathrm{ap}}}
$$

\section{DISCUSSIOK}

First we consider two special cases:

(i) When energetically distributed impurities are not very important, that is, $q N_{0} V_{c} \leqslant N_{d}$ (or $k_{i} V_{c} \leqslant k_{2}$ ): In this case we consider $\cosh x=1+x^{2} / 2$ and $\sinh x \cong x$. Thus

$\cosh ^{-1}\left(\frac{k_{1} V_{c}+k_{2}}{k_{2}}\right)=\cosh ^{-1}\left(1+\frac{k_{1}}{k_{2}} V_{c}\right) \cong\left(\frac{2 k_{1} V_{c}}{k_{2}}\right)^{1 / 2}$

Substituting this value in Eqs. (10) and (13), we get

$$
W=\left(2 V_{c} / k_{2}\right)^{1 / 2}=\left(2 K \epsilon_{0} V_{c} / q N_{d}\right)^{1 / 2}
$$

and

$$
E_{c}(0)=\left(2 q N_{d} V_{c} / K \epsilon_{0}\right)^{1 / 2} .
$$

These are the same results as in intimate Schottky barrier with the only difference that now $V_{c}$ is related to $V_{\text {an }}$ through Eq. (4). In this case $W$ as well as $E_{c}(0)$ are proportional to $W^{1 / 2}$. If the contact current $(B)$ is given through the Schottky effect that is in $I \propto E_{c}(0)^{1 / 2}$, and effective trap density $\left(N_{0} V_{c}+N_{d}\right)$ in the depletion region is less than $10^{18} /$ $\mathrm{cm}^{3}$, then in $I$ will be proportional to $V_{c}^{1 / 4}$ or $\left(V_{\mathrm{ap}}+V_{\mathrm{bi}}\right)^{1 / 4}$. The capacitance in this case is given by

$$
C=\left(2 q N_{d} K \epsilon_{0} / V_{c}\right)^{1 / 2}
$$

or capacitance is inversely proportional to $V_{c}^{1 / 2}$ or $\left(V_{\mathrm{ap}}+V_{\mathrm{bi}}\right)^{1 / 2}$.

(ii) When energetically distributed impurities are predominant, that is, $q N_{0} V_{c} \gg N_{d}$ (or $\left.X_{1} V_{c} \gg K_{2}\right)$ : In this case, we can consider $\cosh x=\sinh x \cong e^{x} / 2$ or $\cosh ^{-1}(x)=\ln (2 x)$. Thus

$$
W=\left(\ell / \sqrt{k_{1}}\right) \ln \left(2 k_{1} V_{c} / k_{2}\right)
$$

and

$$
E_{c}(0)=\left(k_{2} / \sqrt{k_{1}}\right)\left(k_{1} V_{c} / k_{2}\right)=\sqrt{k_{1}} V_{c}
$$

or

$$
E_{\mathrm{c}}(0)=\left[\sqrt{k_{1}} /\left(1+L_{\mathrm{ox}} \sqrt{k_{1}}\right)\right]\left(V_{\mathrm{ap}}+V_{\mathrm{bi}}\right)
$$

In this case $E_{c}(O)$ is proportional to $\left(V_{\text {ap }}+V_{b i}\right)$. lif the current in steady state is through Schotky effect, then In $I \propto\left(V_{\mathrm{ap}}+V_{\mathrm{bi}}\right)^{1 / 2}$ (or $V_{\text {ap }}^{1 / 2}$ if $\left.V_{\text {ap }} \gg V_{\mathrm{bi}}\right)$. As $k_{1} V_{c} / k_{2} \gg 1$, therefore $\ln \left(2 k_{1} V_{c} / k_{2}\right)$ changes very slowly with $V_{c}$ or $V_{\text {ap }}$. Thus the width of the depietion region is almost constant.

The charge inside the depletion region in this case is given by $\left.\left[q^{2} k \epsilon_{0} N_{0}\right)^{1 / 2} V_{c}\right]$. Thus the capacitance of the depletion region is

$$
C=\left(q^{2} k \epsilon_{0} N_{0}\right)^{1 / 2}\left[1+L_{o x}\left(g^{2} N_{0} / k \epsilon_{0}\right)^{1 / 2}\right]^{-1}
$$

i.e., capacitance is almost constant as generally $L_{o x}\left(q^{2} N_{0} / k \epsilon_{0}\right)^{1 / 2} \ll 1$.

For the purpose of illustration of the variation of electrical potential and field, etc., with energetically distributed impurities, we consider the case in which $N_{d}=3 \times 10^{17} \mathrm{im}$ purities $/ \mathrm{cm}^{3}$ and $N_{0}$ varies between $10^{16}$ to $10^{20}$ impurities $\mathrm{cm}^{3} \mathrm{eV}$. The dielectric constant is considered to be 11.7 . The thickness of the oxide layer is taken to be $50 \mathrm{~A}$.

In Fig. 4, we plot the variation of electrical potential within the depletion region for various values of $V_{c}$. In Figs. $4(\mathrm{a}), 4(\mathrm{~b}), 4(\mathrm{c}), 4(\mathrm{~d})$, and $4(\mathrm{e})$, we consider $N_{0}=10^{16}$, $10^{17}, 10^{18}, 10^{19}$, and $10^{20}$ impurities $/ \mathrm{eV} \mathrm{cm}^{3}$, respectively. In Fig. 5, we have plotted the variation of potential in the depletion region for various values of $N_{0}$, considering $V_{c}=3 \mathrm{~V}$. We observe that for comparatively low values of $q N_{0} V_{c}$, the potential is distributed over comparatively long distances for higher values of applied potential and $V_{s}$ is proportional to $x^{2}$ as in the Schottky barrier. For comparatively higher values of $q N_{0} V_{c}$, the potentials is distributed over aimost the same distances for various applied potertials.

In Fig. 6 we plot the variation of electrical charge released in dielectric relaxacion $(Q)$ with applied potential for various values of $N_{0}$. For low values of $q N_{0} V_{c}$ the electrical charge is nearly proportional to $V_{\text {ap }}^{1 / 2}$, while for higher values it is proportional to $V_{\text {ap }}$. In Fig. 7 we give the variation of capacitance with applied potential. For low values of $q N_{0} V_{c}$, capacitance is inversely proportional to $\sqrt{V_{a p}}$, while for higher values it is almost constant.

In Fig. 8 we plot the variation of electrical held within the depietion region for an applied potential equal to $3 \mathrm{~V}$. In Fig. 9 we give the variation of $E_{c}(0)$ with applied potential for various values of $N_{0}$. In case of low $q N_{0} V_{r}, E_{c}(0)$ is proportional to $V_{a p}^{1 / 2}$, while for higher values it is proportional to $V_{\text {ap }}$. In Fig. 10 we plot the variation of thickness of the depletion layer (W) with $V_{a p}$. For lower values of $a N_{0} V_{c}$, W is proportional to $V_{c}^{1 / 2}$, while for higher values, it increases 


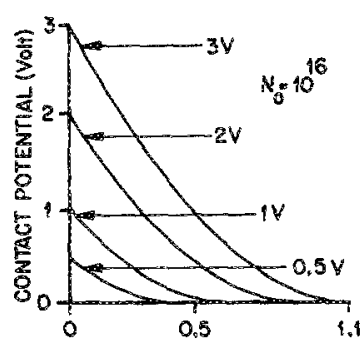

OISTANCE $\times\left(10^{-5} \mathrm{~cm}\right)$

(a)

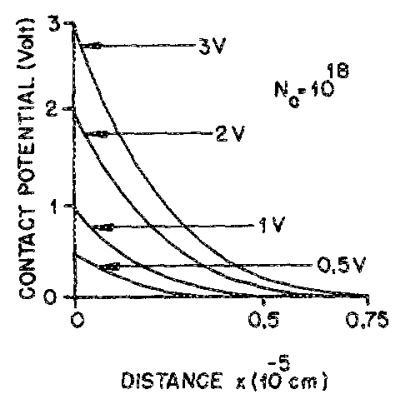

(c)

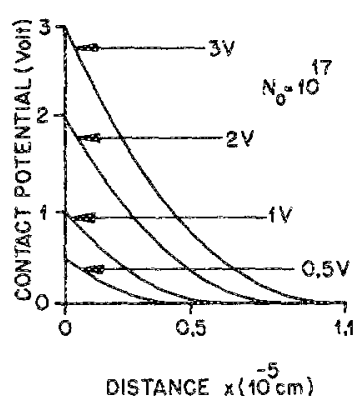

(b)

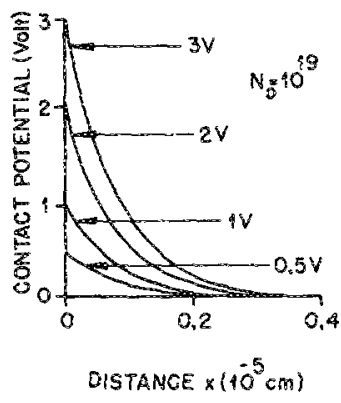

(d)

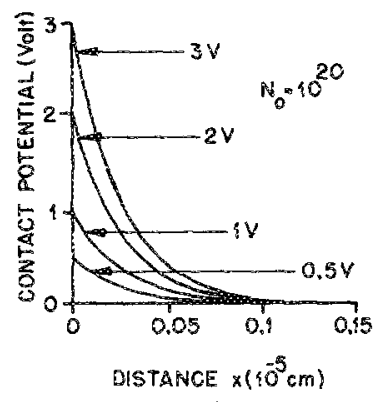

(e)

FIG. 4. Variation of electrical potential inside the depletion region for several values of contact potential $\left(V_{c}\right) \cdot N_{0}$ is $10^{16}, 10^{37}, 10^{18}, 10^{19}$, and $10^{20}$ / $e^{V} \mathrm{~cm}^{3}$ in (a), (b), (c), (d), and (e), respectively

very slowly and can be considered as constant. These are the same results that we obtained analytically.

In conclusion, we can say that when energetically distributed impurities are less important than impurities at a

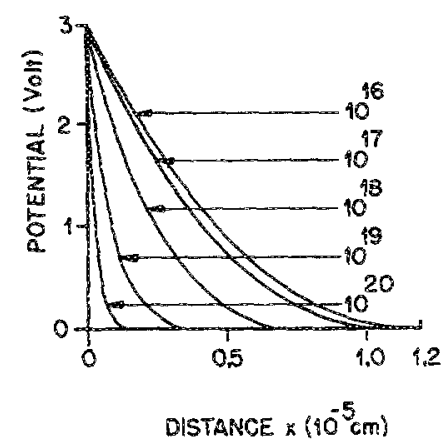

FIG. 5. Variation of electrical potential inside the depletion region for several values of $N_{0} . V_{i}$ is $3 \mathrm{~V}$.

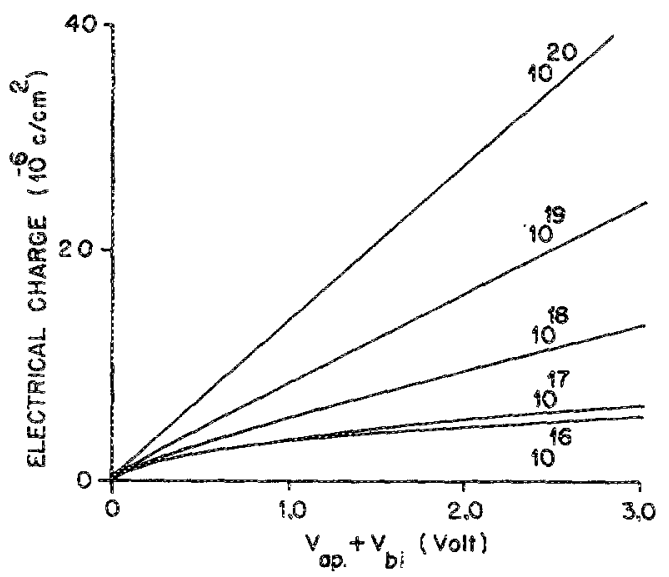

FIG. 6. Variation of electrical charge inside the depletion region with applied potential for several values of $N_{0}$.

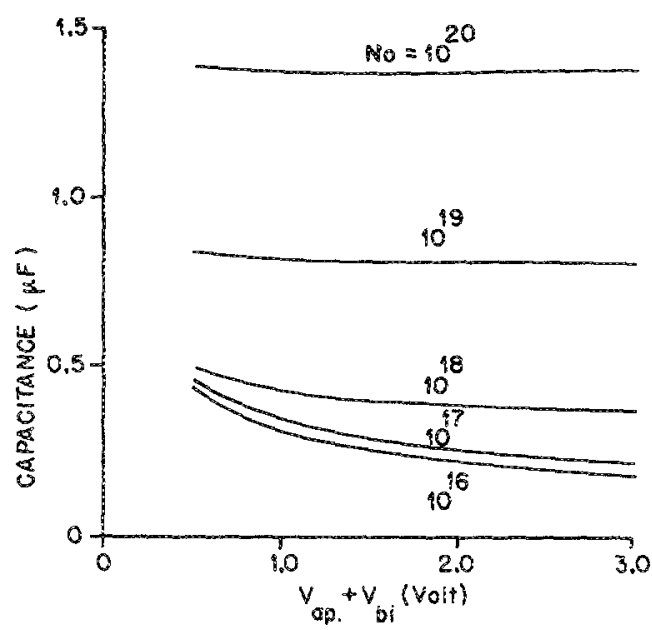

FIG. 7. Variation of capacitance with potential for several values of $N_{0}$

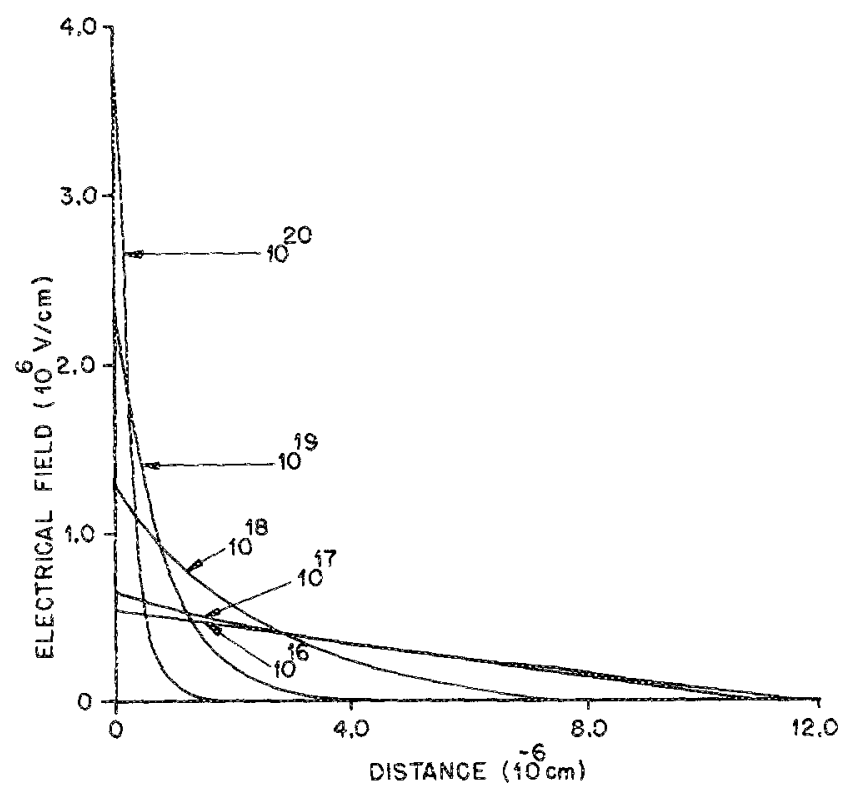

FIG. 8. Variation of electrical field inside the depletion region for several values of $N_{0} . V_{\text {upp }}$ is $3 \mathrm{~V}$. 


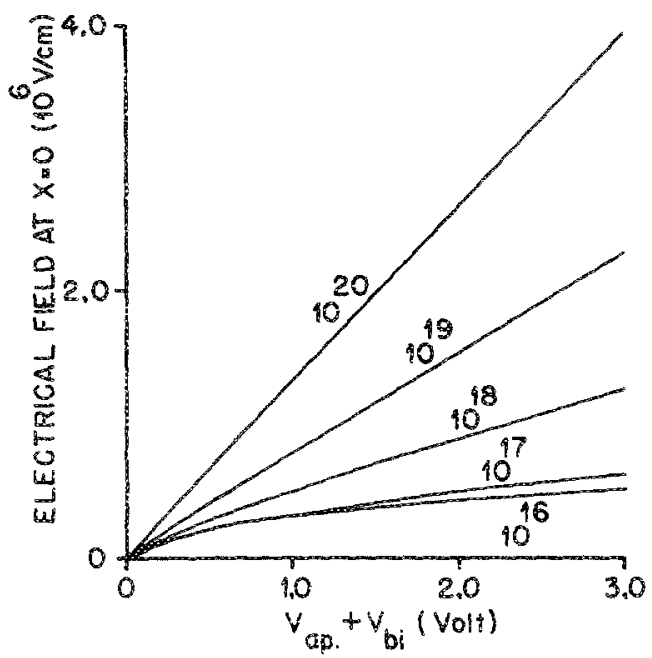

FIG. 9. Variation of electric field at $x=0$ with apphied potential for several values of $N_{0}$

single level, that is, $q N_{0} V_{c} \& N_{d}$, the final results are more or less the same as in an intimate Schottky barrier. The potential fall across the oxide region is very small in comparison to the potential fall across the depletion layer (except when $N_{d} \geqslant 10^{18} / \mathrm{cm}^{3}$ ) and the same results can be used. For $N_{d} \gg$ $10^{18} / \mathrm{cm}^{3}$ we can still use the results, except that we need to use $V_{c}$ instead of $V_{\text {ap }}$. For higher values of $q N_{0} V_{c}$, the final results change significantly. In Table I we compare characteristics in two cases.

The capacitance of the metal-insulator contact is occasionally considered ${ }^{31-33}$ to be constant without considering details of the Schottky barrier. The capacitance of the contact can be considered constant only if energetically distributed impurities are more important in the depletion region.

In MIM structures, characteristics are sometimes considered ${ }^{33-41}$ to be contact limited (Schottky effect) if $\ln I$ is proportional to $V_{\text {ap }}^{1 / 2}$. As per Schottiky effect, $\ln I$ is proportional to $E_{c}(0)^{1 / 2}$. This means that the field is considered uniform throughout the insulator. This is only possible when

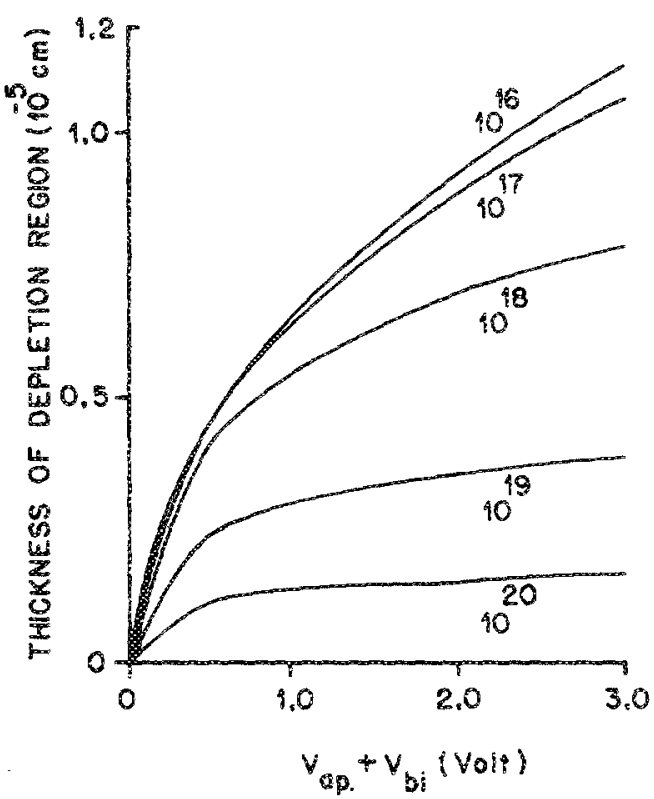

FIG. 10. Variation of the thickness of the depletion region with applied potential for several values of $N_{0}$.

space-charge effects are not present. ${ }^{34}$ In blocking contacts, most of the applied potential appears across the contact and we have a space-charge region. The space-charge effects are important and the electrical field cannot be uniform throughout the insulator. However, if energetically distributed impurities are more important, we still can have $E_{c}(0) \propto V_{\mathrm{ap}}$ and thus $\ln I$ is proportional to $V_{\mathrm{ap}}^{1 / 2}$. In this case distribution of potential is quite complicated and is not uniform. For low density of energetically distributed impurities, we must have $\ln I \propto V_{\varepsilon}^{1 / 4}$ in blocking contacts.

The nonsteady-state characteristics of some metal-insulator-metal structures can be explained better by considering constant capacitance of the space-charge region. ${ }^{42}$ In the present model, the capaciance turns our to be almost constant, when energetically distributed impurities are more important.

TABLE I. Comparison of electrical characteristics of the Schottky barrier.

\begin{tabular}{|c|c|c|c|}
\hline No. & Characteristics & $N_{o} V_{r} \& N_{d}$ & $N_{0} Y_{z} \gg N_{d}$ \\
\hline 1 & $\begin{array}{l}\text { Thickness of the } \\
\text { depletion region }\end{array}$ & $W \propto V_{c}^{1 / 2}$ & $W \propto \ln \left(2 q N_{0} V_{c} / N_{d}\right)$ \\
\hline 2 & $\begin{array}{l}\text { Increase in charge } \\
\text { in depletion region }(Q)\end{array}$ & $Q \propto\left(V_{c}^{1 / 2}-V_{b i}^{1 / 2}\right)$ & $Q \propto r_{u p}$ \\
\hline 3 & $\begin{array}{l}\text { Capacitance of } \\
\text { depletion region }\end{array}$ & $C \cos i^{-1 / 2}$ & $C$ is consiant \\
\hline 4 & $\begin{array}{l}\text { Electrical field at } \\
x=0\end{array}$ & $E_{c}(0) \propto V_{0}^{1 / 2}$ & $E_{c}(0) \propto V_{c}$ or $\left(V_{\mathrm{ap}}+V_{\mathrm{bi}}\right)$ \\
\hline 5 & in $I$ is $V_{c}$ & $\ln I \propto V_{c}^{1 / 4}$ & $\ln I \propto V_{c}^{1 / 2}$ or $\left(V_{a p}+V_{b s}\right)^{1 / 2}$ \\
\hline
\end{tabular}


The results presented here are useful for interpreting the electrical characteristics of MM structures (particularly those of amorphous material) correctly and to have a better understanding of metal-insulator-metal blocking contacts.

"F. Brawn, Progg. Ann. 153, 556 (1874).

${ }^{2}$ K. E. Peterson and D. Adler, J. Appl. Phys. 90,925 (1979).

${ }^{3}$ R. W. Berry, P. M. Hall, and M. T. Harris, Thin Films Technology (Van Nostrand, Princeton, New Jersey, 1968\}.

${ }^{4} \mathrm{H}$. K. Henisch, Rectifying Semiconductor Contacts (Oxford University, London, 1957).

${ }^{5}$ E. H. Rhoderick, Metal-Semiconductor Contacts (Oxford University, London, 1978).

G. Lindau and T. Kendelewicz, C. R. C. Crit. Rev. Solid State Mater. Sci. $13,27(1986)$.

7. D. Cohen and D. V. Lang, Phys. Rev. B 25, 5321 (1982).

${ }^{8}$ F. J. Bryant, J. M. Majio, C. G. Scott, and D. Shaw, Solid State Commun. 63,9(1987).

${ }^{9}$ R. A. Abraham and P. J. Doherty, Philos. Mag. 45, 167 (1982).

${ }^{10}$ A. S. Yapsir, P. Hadizad, T. M. Lu, J. C. Corelli, J. W. Corbett, W. A. Lanford, and H. Bakhre, Phys. Rev. B 37, 8982 (1988).

11. J. Shian and R. H. Bube, Solid-Siate Electron 29, 1153 (1986).

${ }^{12 D}$. V. Lang, J. D. Cohen, and J. P. Harbison, Phys. Rev. B 25, 5285 (1982).

${ }^{13}$ N. F. Mott, Adv. Prys. 16, 49 (1967).

i4. R. Pollack and J. A. Seitchick, Appl. Solid State Sci. Adv. 1, 343 (1969).

${ }^{15}$ C. B. Duke, Torneling in Solids (Academic, New York, 1969). 16w. Schottky, Z. Phys. 113, 367 (1939).

17. Bardeen, Phys. Rev. 71, 717 (1947).

${ }^{28}$ S. G. Lovie and M. L. Cohen, Phys. Rev. B 13, 2461 (1976).

${ }^{19}$ P. Chattopadhyay and A. N. Daw, Solid State Electron, 28, 831 (1985).

${ }^{20}$ S. W. Tarasewicz and C. A. T. Salama, Solid-State Electron 27, 33 (1984).

21. G. Simmons and G.W. Taylor, Phys. Rev. B 5, 553 (1972).

${ }^{22}$ H. M. Gupta and R. J. Van Overstraeten, J. Phys. C 7, 3560 (1974).

${ }^{23}$ M. M. Gupta, J. A ppl. Phys. 48, 3448 (1977).

${ }^{24}$ For example, see S. M. Sze, Physics of Semiconductor Devices (Wiley, Interscience, New York, 1969), p. 371.

25Y. Solomon, T. Dieti, and D. Kaplan, J. Phys. (Paris) 39, 124 (1978).

${ }^{26}$ D. G. Ast and M. H. Brodsky, J. Non-Cryst. Solids 35-36, 611 (1980).

${ }^{27}$ S. J. Fonash. J. Appl. Phys. 47, 3597 (1976).

${ }^{28}$ W. Dabrowski, Phys. Status Solidi A 105, 511 (1988).

${ }^{29}$ K. C. Kao and W. Hwang, Electrical Transport in Solids (Pergamon, OXford, [981).

${ }^{30}$ J. Rybicki and M. Chybicki, Thin Solid Films 149, 261 (1987).

${ }^{34}$ B. L. Timon, Sov. Phys. Sernicond. 7, 163 (1973).

${ }^{32}$ G. P. Srivastava, P. K. Bhatnagar, and S. R. Dharisyal, Solid-State Electron 22, 581 (1979)

${ }^{33}$ C. M. Vodemicharov and M. B. Yodemicharov, Phys. Status Solidi A 57, 483 (1980).

${ }^{34}$ R. I. Frani and J. G. Simmons, 3. Appi. Phys. 38, 832 (1967).

${ }^{35}$ G. Lengyel, I. Appl. Phys. 37, 807 (1966).

${ }^{36}$ I. T. Johansen, J. Appl. Phys. 37, 499 (1966).

37T. E. Haltman, J. C. Blair, and R. Bauer, J. Appl, Phys. 37, 2468 (1966).

${ }^{38}$ J. C. Schung, A. C. Lilly, Ir., and M. A. Lowitz, Phys. Rev. B 1, 4811 (1970).

${ }^{39}$ A. C. Lilly, Jr. and J. R. McDowell, J. Appl. Phys. 39, 141 (1968).

${ }^{40}$ P. L. Young, J. Appl. Phys. 47, 242 (1976).

4'D. M. Taylor and T. J. Lewis, J. Phys. D 4, 1346 (1971).

${ }^{42}$ H. M. Gupta, Appl. Prys. Lett. 33, 778 (1978). 